\title{
PAPER \\ Multiple-Symbol Differential Detection for Unitary Space-Time-Frequency Coding
}

\author{
Ziyan JIA $^{\dagger a)}$, Student Member, Shiro HANDA ${ }^{\dagger}$, Fumihito SASAMORI ${ }^{\dagger}$, and Shinjiro OSHITA ${ }^{\dagger}$, Members
}

\begin{abstract}
SUMMARY In this paper, multiple-symbol differential detection (MSDD) is applied to the differential unitary space-time-frequency coding (DUSTFC) scheme over frequency selective fading multiple-input multiple-output (MIMO) channels. The motivation of applying MSDD is to compensate for the performance loss of conventional (two-symbol observation) differential detection comparing with coherent detection, by extending the observation interval and considering the fading autocorrelations. Since the differential coding of DUSTFC can be performed in time or frequency domain, both the time-domain and frequency-domain MSDD are investigated. After calculating the frequency-domain fading autocorrelation, the decision metrics of MSDD considering appropriate fading autocorrelations are derived in time and frequency domain respectively. Bit error rate (BER) performances of the two kinds of MSDD are analyzed by computer simulations. Simulation results demonstrate that a considerable performance gain can be got by applying MSDD in both cases, and the transmit diversity gain can also be enhanced by applying MSDD. So that it is proved that full advantage of transmit diversity with DUSTFC can be taken by applying MSDD.

key words: multiple-symbol differential detection, differential unitary space-time-frequency coding, fading autocorrelation, OFDM, frequency selective fading, MIMO
\end{abstract}

\section{Introduction}

Differential space-time modulation (DSTM) [1]-[3] is an easily implemented and attractive transmit diversity method with multiple transmit antennas in flat fading channels. With DSTM, no channel state information (CSI) is needed at both transmitter and receiver. This makes DSTM very competitive when fading channels change so rapidly that the CSI which is necessary for coherent detection cannot be got reliably.

In order to utilize DSTM over frequency selective fading channels, orthogonal frequency division multiplexing (OFDM) [4], which converts the frequency selective channel into a set of flat fading subchannels, was combined with DSTM [5], [6]. In frequency selective channels, frequency (multipath) diversity is an extra diversity resource besides space diversity. Frequency diversity can be achieved by simply applying the subcarrier grouping [7] method. With subcarrier grouping, the OFDM carriers are divided into groups of subcarriers, and replicas of the same signal are transmitted from different subcarriers in a same subcarrier group to provide transmit diversity gain. To achieve a higher

\footnotetext{
Manuscript received December 8, 2008.

Manuscript revised August 15, 2009.

${ }^{\dagger}$ The authors are with the Faculty of Engineering, Shinshu University, Nagano-shi, 380-8553 Japan.

a) E-mail: jiaziyan@oshan.shinshu-u.ac.jp

DOI: 10.1587/transcom.E93.B.90
}

transmit diversity order, a differential unitary space-timefrequency coding (DUSTFC) scheme was proposed in [5] by incorporating subcarrier grouping and differential unitary space-time modulation (DUSTM) [2], [3].

It is well known that conventional (two-symbol observation) differential detection has a considerable performance loss comparing with coherent detection. Multiplesymbol differential detection (MSDD) [8], which was first presented for differential phase shift keying (DPSK), is effective to compensate for such a performance loss. The basic idea of MSDD is to extend the observation interval to more than two symbols and make a joint decision on those several symbols simultaneously, instead of symbolby-symbol detection as in conventional differential detection. Furthermore, in continuous fading channels, observing multiple successive symbols makes considering fading autocorrelations to enhance the system performance possible.

Since the differential coding of DUSTFC can be performed in either time or frequency domain, MSDD receivers for both cases are investigated. In some works such as [9], [10] and [11], the time-domain fading autocorrelation has been taken into account for time-domain MSDD. In this paper, the fading autocorrelation is considered for MSDD in both the time-domain and frequency-domain cases. Since the existing frequency-domain fading autocorrelation function [12] between subcarriers is not fit for digital OFDM systems when the separation is big (as shown in Sect. 3.5 below), we recalculate the frequency-domain fading autocorrelation function between OFDM subcarriers in frequency selective fading channels in this paper. Decision metrics of time-domain and frequency-domain MSDD are derived by applying the well known time-domain fading autocorrelation in [13] and the calculated frequency-domain fading autocorrelation respectively.

Bit error rate (BER) performances of the time and frequency domain MSDD for DUSTFC in fast frequency selective Rayleigh fading channels are analyzed by computer simulations. Simulation results demonstrate that in both time and frequency domain, after considering the fading autocorrelation, extending the observation interval can lead to a considerable performance gain as well as an enhanced diversity gain. So that full advantage of the improved diversity order provided by DUSTFC can be taken.

The rest of this paper is organized as follows. In Sect. 2, we introduce the system model. In Sect. 3, DUSTFC is reviewed. The decision metrics of MSDD are derived in 
Sect. 4. The simulation results and related discussion are given in Sect. 5 and finally the conclusion is in Sect. 6.

\section{System Model}

We consider a wireless communication system with $N_{t}$ transmit antennas and $N_{r}$ receive antennas. The channel between each transmit and receive antenna is assumed to be time-varying frequency selective multipath Rayleigh fading with channel order $L$. OFDM is employed at each antenna, and the number of OFDM subcarriers is $N_{c}$. The transmitted signals are grouped in blocks of size $N_{x}$ at time domain, and we assume $N_{x}=N_{t}$ in this paper. At time $N_{t} \tau+k$, $0 \leq k \leq N_{t}-1$, a symbol $s_{\mu}^{p}\left(N_{t} \tau+k\right)$ is transmitted by the $\mu$ th antenna on subcarrier $p$. Assuming ideal timing and carrier synchronization, and a cyclic prefix $(\mathrm{CP})$ length greater than channel order $L$, after FFT processing, the received signal of $s_{\mu}^{p}\left(N_{t} \tau+k\right)$ at receive antenna $v$ can be expressed as

$$
\begin{aligned}
& y_{\nu}^{p}\left(N_{t} \tau+k\right) \\
& =\sum_{\mu=1}^{N_{t}} H_{\mu \nu}^{p}\left(N_{t} \tau+k\right) s_{\mu}^{p}\left(N_{t} \tau+k\right)+n_{\nu}^{p}\left(N_{t} \tau+k\right),
\end{aligned}
$$

where

$$
H_{\mu \nu}^{p}(t)=\sum_{l=0}^{L} h_{\mu \nu}^{l}(t) \exp \left(-j 2 \pi l p / N_{c}\right)
$$

is the subchannel gain between the $\mu$ th transmit antenna and the $v$ th receive antenna at time $t$ on the $p$ th subcarrier. $h_{\mu v}^{l}(t)$, which is a complex Gaussian random variable with mean zero and variance $\sigma_{h}^{2}(l)$, is the discrete-time baseband equivalent impulse response of $l$ th tap. And as usual $h_{\mu \nu}^{l}(t)$ is assumed to be uncorrelated with $h_{\mu^{\prime} v^{\prime}}^{l^{\prime}}(t)$ when $l \neq l^{\prime}$ and/or $\mu \neq \mu^{\prime}$ and/or $v \neq v^{\prime}$. It is easy to calculate that the mean and variance of $H_{\mu \nu}^{p}(t)$ are zero and $\sigma_{h}^{2}=\sum_{l=0}^{L} \sigma_{h}^{2}(l)$ respectively. $n_{v}^{p}(t)$ is additive complex Gaussian noise with zero mean and variance $\sigma_{n}^{2}$ at receive antenna $v$, and $n_{\nu}^{p}(t)$ is uncorrelated with $n_{v^{\prime}}^{p^{\prime}}\left(t^{\prime}\right)$ when $p \neq p^{\prime}$ and/or $v \neq v^{\prime}$ and/or $t \neq t^{\prime}$.

\section{Differential Unitary Space-Time-Frequency Coding}

\subsection{Space-Time Coding}

Define $N_{t} \times N_{t}$ space-time code matrix of transmitted symbols at $\tau$ th time block on subcarrier $p$ as $\mathbf{S}^{p}(\tau)$ with $\left[\mathbf{S}^{p}(\tau)\right]_{k \mu}=s_{\mu}^{p}\left(N_{t} \tau+k\right)$, where $[\cdot]_{i j}$ denotes the $i$, jth element of a matrix, and assume a block fading environment i.e.

$$
\begin{aligned}
H_{\mu \nu}^{p}\left(N_{t} \tau+k\right)= & H_{\mu \nu}^{p}\left(N_{t} \tau+k^{\prime}\right)=H_{\mu \nu}^{p}\left(N_{t} \tau\right) \\
& 0 \leq k \leq k^{\prime} \leq N_{t}-1
\end{aligned}
$$

We can recast (1) to an equivalent matrix form as

$$
\mathbf{Y}^{p}(\tau)=\mathbf{S}^{p}(\tau) \mathbf{H}^{p}(\tau)+\mathbf{N}^{p}(\tau)
$$

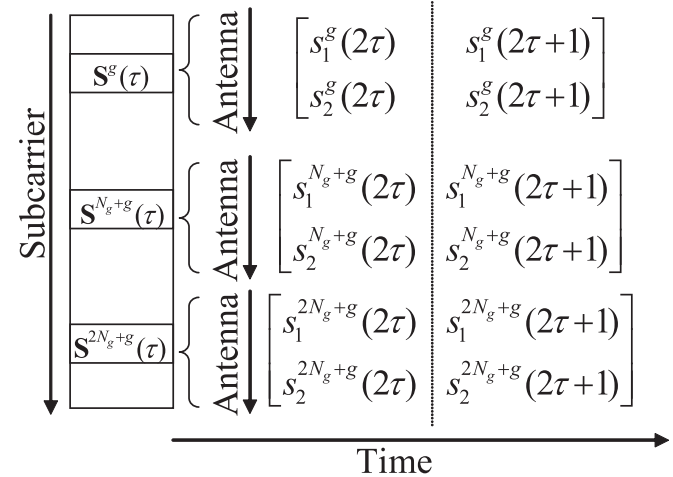

Fig. 1 An example of the structure of a DUSTFC codeword $\mathbf{S}_{g}(\tau)$. $(M+$ $1=3, N_{t}=2$ ).

where $\mathbf{Y}^{p}(\tau), \mathbf{H}^{p}(\tau)$ and $\mathbf{N}^{p}(\tau)$ are $N_{t} \times N_{r}$ matrices with $\left[\mathbf{Y}^{p}(\tau)\right]_{k v}=y_{\nu}^{p}\left(N_{t} \tau+k\right),\left[\mathbf{H}^{p}(\tau)\right]_{\mu \nu}=H_{\mu \nu}^{p}\left(N_{t} \tau+k\right)$ and $\left[\mathbf{N}^{p}(\tau)\right]_{k v}=n_{v}^{p}\left(N_{t} \tau+k\right)$ respectively.

\subsection{Subcarrier Grouping}

To achieve a higher diversity order with low complexity design, subcarrier grouping is utilized in DUSTFC. With subcarrier grouping, the total $N_{c}$ subcarriers are partitioned into $N_{g}$ groups of subcarriers and each group has $M+1$ subcarriers, i.e.

$$
N_{c}=(M+1) \times N_{g} .
$$

Equispaced subcarrier grouping scheme is used and therefore the $(m+1)$ th subcarrier in $g$ th group is subcarrier $m N_{g}+g, 0 \leq m \leq M$.

The received signals of all the subcarriers in group $g$ can be rewritten as

$$
\mathbf{Y}_{g}(\tau)=\mathbf{S}_{g}(\tau) \mathbf{H}_{g}(\tau)+\mathbf{N}_{g}(\tau)
$$

where $\mathbf{Y}_{g}(\tau), \mathbf{H}_{g}(\tau)$ and $\mathbf{N}_{g}(\tau)$ are $(M+1)$ th order vectors of $N_{t} \times N_{r}$ matrices, and $\left[\mathbf{Y}_{g}(\tau)\right]_{m+1}=\mathbf{Y}^{m N_{g}+g}(\tau),\left[\mathbf{H}_{g}(\tau)\right]_{m+1}=$ $\mathbf{H}^{m N_{g}+g}(\tau)$ and $\left[\mathbf{N}_{g}(\tau)\right]_{m+1}=\mathbf{N}^{m N_{g}+g}(\tau)$ respectively. Here $[\cdot]_{i}$ denotes the $i$ th element of a vector. DUSTFC codeword $\mathbf{S}_{g}(\tau)$ is an $(M+1) \times(M+1)$ block diagonal matrix of $N_{t} \times N_{t}$ matrices, and $\left[\mathbf{S}_{g}(\tau)\right]_{(m+1)(m+1)}=\mathbf{S}^{m N_{g}+g}(\tau)$. An example of the structure of $\mathbf{S}_{g}(\tau)$ is shown in Fig. 1.

\subsection{Differential Encoding}

Let $G$ be a finite group of $(M+1) N_{t} \times(M+1) N_{t}$ unitary matrices with $V$ elements. For a data rate of $R$ bits per subcarrier use, we need to design $G$ with cardinality $V=2^{R(M+1) N_{t}}$. It is known that the differential encoding of DUSTFC can be performed in both time and frequency domain [6]. In time domain, data are carried by two consecutive time blocks at the same subcarrier group. The transmitted codeword from the $g$ th group can be got by

$$
\mathbf{S}_{g}(\tau)= \begin{cases}\mathbf{V}_{g}(\tau) \mathbf{S}_{g}(\tau-1), & \tau \geqslant 1 \\ \mathbf{I}_{(M+1) N_{t}}, & \tau=0\end{cases}
$$


where the $(M+1) N_{t} \times(M+1) N_{t}$ unitary matrix $\mathbf{V}_{g}(\tau) \in G$ conveys the transmit information and $\mathbf{I}_{M}$ is an $M \times M$ identity matrix. Note that $\mathbf{S}_{g}(\tau)$ is unitary and the transmit power is constant, because $\mathbf{V}_{g}(\tau)$ is unitary.

Similarly, in frequency domain, data are carried by two neighboring subcarrier groups at the same time block. The transmitted codeword at time block $\tau$ is

$$
\mathbf{S}_{g}(\tau)= \begin{cases}\mathbf{V}_{g}(\tau) \mathbf{S}_{g-1}(\tau), & g \geqslant 1 \\ \mathbf{I}_{(M+1) N_{t}}, & g=0\end{cases}
$$

\subsection{Constellations of DUSTFC}

Both diagonal and nondiagonal constellations can be used as the constellations of DUSTFC as in [6]. In [6], it is proved that both diagonal and nondiagonal constellations have socalled maximum diversity, and yield excellent performance in time-unvarying frequency selective fading channels. But since the channel is required to be approximately constant during transmitting a nondiagonal matrix [14], nondiagonal constellations are not fit for time-varying fading channels very well, especially when the fading is very fast. Because we consider the time-varying fading channels in this paper, we prefer to choose the diagonal constellations. However, as shown later in Sect. 4, nondiagonal constellations can also be employed for the proposed system easily.

\subsubsection{Abelian Group (Diagonal) Constellations}

For diagonal constellations, the group $G$ is assumed to be Abelian. As shown in [5], the elements of $G$ are diagonal matrices, and at rate $R$ the optimal Abelian group $G$ of DUSTFC in the sense of maximizing the coding advantage is the optimal Abelian group for a flat fading channel with $T=(M+1) N_{t}$ transmit antennas with the same rate $R$. Therefore, the $a$ th element of $G$ is

$$
G_{a}=\left[\begin{array}{ccc}
e^{j \frac{2 \pi}{V} u_{1} a} & 0 & \cdots \\
0 & \ddots & 0 \\
0 & \ldots & e^{j \frac{2 \pi}{V} u_{T} a}
\end{array}\right]
$$

where $u_{m} \in\{0, \ldots, V-1\}, 1 \leq m \leq T$. And the optimal design of $u_{1}, \ldots, u_{T}$ can be found by satisfying

$$
\arg \max _{0<=u_{1}, \ldots, u_{T}<=V-1} \min _{a=1, \ldots, V-1}\left|\prod_{m=1}^{T} \sin \left(\pi u_{m} a / V\right)\right|^{\frac{1}{T}}
$$

By $\mathbf{V}_{g}(\tau) \in G$, (7) and (8), we know that $\mathbf{V}_{g}(\tau)$ and $\mathbf{S}_{g}(\tau)$ are diagonal matrices. Obviously, each submatrix $\mathbf{S}^{m N_{g}+g}(\tau)$ of $\mathbf{S}_{g}(\tau)$, is also a diagonal matrix. As shown is Fig. 1, when $\mathbf{S}^{m N_{g}+g}(\tau)$ is a diagonal matrix, each transmit antenna is acted only once, to transmit signals from all the subcarriers simultaneously, during transmitting a DUSTFC codeword.

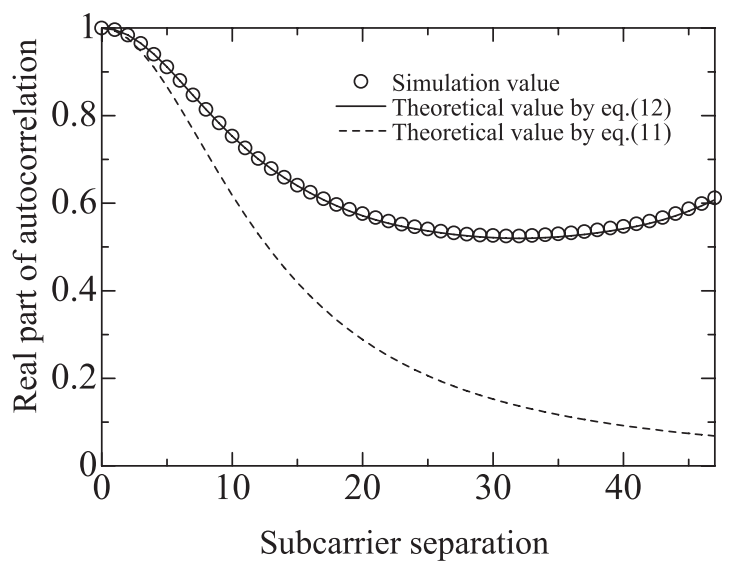

Fig. 2 The comparation of frequency-domain fading autocorrelation (real part). $\left(\sigma_{\tau} / T_{s}=0.01, T_{g} / T_{d}=0.25\right)$

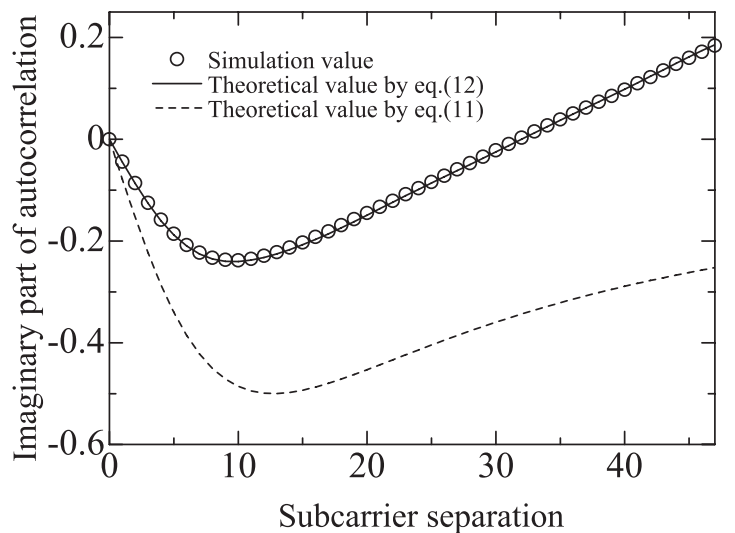

Fig. 3 The comparation of frequency-domain fading autocorrelation (imaginary part). $\left(\sigma_{\tau} / T_{s}=0.01, T_{q} / T_{d}=0.25\right)$.

\subsection{Frequency-Domain Fading Autocorrelation}

In [12], the frequency-domain fading autocorrelation function between OFDM subcarriers $p$ and $p^{\prime}$ is derived by the Fourier transform of the continuous exponential power delay profile as

$$
\rho_{F^{\prime}}\left(p-p^{\prime}\right)=\frac{1}{1+j 2 \pi\left(1+\frac{T_{g}}{T_{d}}\right)\left(p-p^{\prime}\right) \frac{\sigma_{\tau}}{T_{s}}}
$$

where $\sigma_{\tau}$ is the root mean square delay spread and $T_{s}(=$ $T_{d}+T_{q}$ ) is an OFDM symbol period, which is composed of a CP period $T_{g}$ and a data symbol period $T_{d}$. In Figs. 2 and 3 we can observe that when the subcarrier separation $p-p^{\prime}$ is small, the derived fading autocorrelation by (11) is very approximate to the simulation value. Since in [12], the authors are mainly focused on the situation when $p-p^{\prime}=1$, (11) is accurate enough.

But because we want to apply MSDD in this paper, fading autocorrelations between large subcarrier separation have to be taken into account. In Figs. 2 and 3, it can be observed that when $p-p^{\prime}$ is big, comparing with the simu- 
lation value, the derived fading autocorrelation by (11) becomes too rough to be used. Therefore, in this paper we rederive the frequency-domain fading autocorrelation function between OFDM subcarriers as follows:

$$
\begin{aligned}
\rho_{F}\left(p-p^{\prime}\right)=E\left\{H_{\mu \nu}^{p}(t) H_{\mu \nu}^{p^{\prime} *}(t)\right\} \\
=E\left[\sum_{l=0}^{L} h_{\mu \nu}^{l}(t) \exp \left(-j 2 \pi l p / N_{c}\right)\right. \\
\left.\quad \cdot \sum_{l=0}^{L} h_{\mu \nu}^{l *}(t) \exp \left(j 2 \pi l p^{\prime} / N_{c}\right)\right] \\
=\sum_{l=0}^{L} E\left[h_{\mu \nu}^{l}(t) h_{\mu \nu}^{l *}(t)\right] \exp \left[-j 2 \pi l\left(p-p^{\prime}\right) / N_{c}\right] \\
=\sum_{l=0}^{L} \sigma_{h}^{2}(l) \exp \left[-j 2 \pi l\left(p-p^{\prime}\right) / N_{c}\right]
\end{aligned}
$$

where the superscript ' $*$ ' denotes the complex conjugation, and $\sigma_{h}^{2}(l)$ is decided by $\sigma_{\tau} / T_{s}$ and $T_{g} / T_{d}$. And it can be observed that, with any subcarrier separation, the rederived fading autocorrelation by (12) fits the simulation value very well.

\section{Multiple-Symbol Differential Detection}

Here we assume the observation interval of MSDD consists of $N$ blocks. Since the differential encoding can be performed in time or frequency domain, MSDD receivers for DUSTFC are investigated in time and frequency domain by considering the appropriate fading autocorrelations respectively.

\subsection{Time-Domain Multiple-Symbol Differential Detection}

When time-domain differential encoding is performed, the received sequence blocks from time $\tau-N+1$ to time $\tau$ of group $g$ can be expressed as

$$
\overline{\mathbf{Y}}_{g}=\overline{\mathbf{S}}_{g} \overline{\mathbf{H}}_{g}+\overline{\mathbf{N}}_{g}
$$

where

$$
\begin{aligned}
\overline{\mathbf{Y}}_{g} & =\left[\mathbf{Y}_{g}^{T}(\tau) \ldots \mathbf{Y}_{g}^{T}(\tau-N+1)\right]^{T} \\
\overline{\mathbf{S}}_{g} & =\operatorname{diag}\left\{\mathbf{S}_{g}(\tau), \ldots, \mathbf{S}_{g}(\tau-N+1)\right\} \\
\overline{\mathbf{H}}_{g} & =\left[\mathbf{H}_{g}^{T}(\tau) \ldots \mathbf{H}_{g}^{T}(\tau-N+1)\right]^{T} \\
\overline{\mathbf{N}}_{g} & =\left[\mathbf{N}_{g}^{T}(\tau) \ldots \mathbf{N}_{g}^{T}(\tau-N+1)\right]^{T}
\end{aligned}
$$

and the superscript ' $T$ ' denotes the transpose operation.

When the receiver has no CSI, the probability density function (PDF) of received signal $\overline{\mathbf{Y}}_{g}$ conditioned on $\overline{\mathbf{S}}_{g}$ is given by

$$
p\left(\overline{\mathbf{Y}}_{g} \mid \overline{\mathbf{S}}_{g}\right)=\frac{\exp \left\{-\operatorname{tr}\left(\overline{\mathbf{Y}}_{g}^{H} \Lambda^{-1} \overline{\mathbf{Y}}_{g}\right)\right\}}{\left(\pi^{N N_{t}(M+1)} \operatorname{det} \Lambda\right)^{N_{r}}}
$$

where ' $t r$ ' denotes the trace function.

$$
\Lambda=E\left[\overline{\mathbf{Y}}_{g} \overline{\mathbf{Y}}_{g}^{H} \mid \overline{\mathbf{S}}_{g}\right]
$$

is the covariance matrix of $\overline{\mathbf{Y}}_{g}$. ' $E$ ' denotes expectation and the superscript ' $H$ ' denotes the Hermitian operation (transpose conjugation). Since $\overline{\mathbf{H}}_{g}$ is independent from $\overline{\mathbf{N}}_{g}, \Lambda$ can be rewritten into

$$
\begin{aligned}
\Lambda & =E\left[\left(\overline{\mathbf{S}}_{g} \overline{\mathbf{H}}_{g}+\overline{\mathbf{N}}_{g}\right)\left(\overline{\mathbf{S}}_{g} \overline{\mathbf{H}}_{g}+\overline{\mathbf{N}}_{g}\right)^{H}\right] \\
& =E\left[\overline{\mathbf{S}}_{g} \overline{\mathbf{H}}_{g} \overline{\mathbf{H}}_{g}^{H} \overline{\mathbf{S}}_{g}^{H}+\overline{\mathbf{N}}_{g} \overline{\mathbf{N}}_{g}^{H}\right] \\
& =\overline{\mathbf{S}}_{g} E\left(\overline{\mathbf{H}}_{g} \overline{\mathbf{H}}_{g}^{H}\right) \overline{\mathbf{S}}_{g}^{H}+N_{r} \sigma_{n}^{2} \mathbf{I}_{N N_{t}(M+1)}
\end{aligned}
$$

Because $h_{\mu \nu}^{l}(t)$ is assumed to be spatial and multipath uncorrelated, we have

$$
\begin{aligned}
& E\left\{h_{\mu \nu}^{l}(t) h_{\mu^{\prime} v^{\prime}}^{l^{\prime} *}\left(t^{\prime}\right)\right\}=0 \\
& \quad\left(l \neq l^{\prime} \text { and/or } \mu \neq \mu^{\prime} \text { and/or } v \neq v^{\prime}\right)
\end{aligned}
$$

And the time-domain fading autocorrelation can be modeled as [13]

$$
\rho_{T}^{l}\left(t^{\prime}-t\right)=E\left\{h_{\mu \nu}^{l}(t) h_{\mu \nu}^{l *}\left(t^{\prime}\right)\right\}=\sigma_{h}^{2}(l) J_{0}\left(2 \pi f_{D} T_{s}\left(t^{\prime}-t\right)\right)
$$

where $f_{D}$ is the maximum Doppler frequency, and $J_{0}(\cdot)$ is the zeroth order Bessel function of the first kind. Set $\rho_{T}\left(t^{\prime}-\right.$ $t)=J_{0}\left(2 \pi f_{D} T_{s}\left(t^{\prime}-t\right)\right)$. Therefore

$$
\begin{gathered}
E\left\{H_{\mu \nu}^{p}(t) H_{\mu^{\prime} v^{\prime}}^{p *}\left(t^{\prime}\right)\right\}=0 \quad\left(\mu \neq \mu^{\prime} \text { and/or } v \neq v^{\prime}\right), \\
E\left\{H_{\mu \nu}^{p}(t) H_{\mu \nu}^{p *}\left(t^{\prime}\right)\right\}=\sum_{l=0}^{L} \sigma_{h}^{2}(l) J_{0}\left(2 \pi f_{D} T_{s}\left(t^{\prime}-t\right)\right) \\
=\sigma_{h}^{2} J_{0}\left(2 \pi f_{D} T_{s}\left(t^{\prime}-t\right)\right) \\
=\sigma_{h}^{2} \rho_{T}\left(t^{\prime}-t\right)
\end{gathered}
$$

and

$$
E\left[\overline{\mathbf{H}}_{g} \overline{\mathbf{H}}_{g}^{H}\right]=N_{r} \sigma_{h}^{2}\left(\Gamma_{T} \otimes \mathbf{I}_{N_{t}(M+1)}\right)
$$

where $\otimes$ denotes the Kronecker product and $\Gamma_{T}$ is an $N \times N$ matrix with $\left[\Gamma_{T}\right]_{i j}=\rho_{T}\left(N_{t}(i-j)\right)$. And

$$
\Lambda=N_{r} \sigma_{h}^{2} \overline{\mathbf{S}}_{g}\left(\Gamma_{T} \otimes \mathbf{I}_{N_{t}(M+1)}\right) \overline{\mathbf{S}}_{g}^{H}+N_{r} \sigma_{n}^{2} \mathbf{I}_{N N_{t}(M+1)}
$$

To calculate the inverse matrix of $\Lambda$, we can use the matrix inversion lemma

$$
(\mathbf{B}+\mathbf{C F D})^{-1}=\mathbf{B}^{-1}-\mathbf{B}^{-1} \mathbf{C}\left(\mathbf{F}^{-1}+\mathbf{D B}^{-1} \mathbf{C}\right)^{-1} \mathbf{D B}^{-1} .
$$

Set $\mathbf{B}=N_{r} \sigma_{n}^{2} \mathbf{I}_{N N_{t}(M+1)}, \mathbf{C}=\overline{\mathbf{S}}_{g}\left(\Gamma_{T} \otimes \mathbf{I}_{N_{t}(M+1)}\right), \mathbf{F}=$ $N_{r} \sigma_{h}^{2} \mathbf{I}_{N N_{t}(M+1)}$ and $\mathbf{D}=\overline{\mathbf{S}}_{g}^{H}$, following a similar process as in [11], we can finally get

$$
\Lambda^{-1}=\frac{1}{N_{r} \sigma_{h}^{2}} \overline{\mathbf{S}}_{g}\left[\left(\frac{\sigma_{n}^{2}}{\sigma_{h}^{2}} \mathbf{I}_{N}+\Gamma_{T}\right)^{-1} \otimes \mathbf{I}_{N_{t}(M+1)}\right] \overline{\mathbf{S}}_{g}^{H} .
$$

Since the natural logarithm is a monotonically increasing function of its argument, maximizing $p\left(\overline{\mathbf{Y}}_{g} \mid \overline{\mathbf{S}}_{g}\right)$ is equivalent to maximizing $\ln p\left(\overline{\mathbf{Y}}_{g} \mid \overline{\mathbf{S}}_{g}\right)$. After taking logarithm of (14) and ignoring the terms which are irrelevant to $\overline{\mathbf{S}}_{g}$, we can get the decision metric of time-domain MSDD as 


$$
\eta^{\prime}=-\operatorname{tr}\left\{\overline{\mathbf{Y}}_{g}^{H} \overline{\mathbf{S}}_{g}\left[\left(\frac{\sigma_{n}^{2}}{\sigma_{h}^{2}} \mathbf{I}_{N}+\Gamma_{T}\right)^{-1} \otimes \mathbf{I}_{N_{t}(M+1)}\right] \overline{\mathbf{S}}_{g}^{H} \overline{\mathbf{Y}}_{g}\right\}
$$

Define

$$
t_{i j}=\left[-\left(\frac{\sigma_{n}^{2}}{\sigma_{h}^{2}} \mathbf{I}_{N}+\Gamma_{T}\right)^{-1}\right]_{i j},
$$

so that the decision metric for diagonal constellations can be finally simplified into

$$
\begin{aligned}
\eta= & \mathfrak{R}\left\{\sum_{n=0}^{M} \sum_{\mu=0}^{N_{t}-1} \sum_{v=0}^{N_{r}-1} \sum_{i=0}^{N-1} \sum_{k=i+1}^{N-1} t_{i k} \prod_{m=i}^{k-1} e^{j \frac{2 \pi u_{\mu M+n} a_{m}}{2^{R(M+1) N_{t}}}}\right. \\
& \left.\cdot y_{v}{ }^{n N_{g}+g *}\left(N_{t}(\tau-i)+\mu\right) y_{v}^{n N_{g}+g}\left(N_{t}(\tau-k)+\mu\right)\right\}
\end{aligned}
$$

where ' $\mathfrak{R}$ ' denotes the the real part of a complex number,

$$
\begin{aligned}
& s_{\mu}{ }^{n N_{g}+g}\left(N_{t}(\tau-i)+\mu\right) s_{\mu}{ }^{n N_{g}+g *}\left(N_{t}(\tau-k)+\mu\right) \\
& =\prod_{m=i}^{k-1} \exp \left(j \frac{2 \pi u_{\mu M+n} a_{m}}{2^{R(M+1) N_{t}}}\right),
\end{aligned}
$$

and $a_{m}$ denotes the transmitted information by a pair of adjacent DUSTFC codewords. Define the sequence of information transmitted in an MSDD observation interval as

$$
\mathbf{A}=\left\{a_{0}, \ldots, a_{N-2}\right\} .
$$

Then $\hat{\mathbf{A}}$, which is an estimation of $\mathbf{A}$, is determined, if (26) is maximized.

4.2 Frequency-Domain Multiple-Symbol Differential Detection

When frequency-domain differential encoding is performed, the received sequence blocks from group $g-N+1$ to group $g$ at time $\tau$ can be expressed as

$$
\overline{\mathbf{Y}}(\tau)=\overline{\mathbf{S}}(\tau) \overline{\mathbf{H}}(\tau)+\overline{\mathbf{N}}(\tau)
$$

where

$$
\begin{aligned}
& \overline{\mathbf{Y}}(\tau)=\left[\mathbf{Y}_{g}^{T}(\tau) \ldots \mathbf{Y}_{g-N+1}^{T}(\tau)\right]^{T} \\
& \overline{\mathbf{S}}(\tau)=\operatorname{diag}\left\{\mathbf{S}_{g}(\tau), \ldots, \mathbf{S}_{g-N+1}(\tau)\right\} \\
& \overline{\mathbf{H}}(\tau)=\left[\mathbf{H}_{g}^{T}(\tau) \ldots \mathbf{H}_{g-N+1}^{T}(\tau)\right]^{T} \\
& \overline{\mathbf{N}}(\tau)=\left[\mathbf{N}_{g}^{T}(\tau) \ldots \mathbf{N}_{g-N+1}^{T}(\tau)\right]^{T}
\end{aligned}
$$

When the receiver has no CSI, the PDF of received signal $\overline{\mathbf{Y}}(\tau)$ conditioned on $\overline{\mathbf{S}}(\tau)$ is given by

$$
p(\overline{\mathbf{Y}}(\tau) \mid \overline{\mathbf{S}}(\tau))=\frac{\exp \left\{-\operatorname{tr}\left(\overline{\mathbf{Y}}(\tau)^{H} \Lambda^{-1} \overline{\mathbf{Y}}(\tau)\right)\right\}}{\left(\pi^{N N_{t}(M+1)} \operatorname{det} \Lambda\right)^{N_{r}}}
$$

where similar to the time-domain case, the covariance matrix $\Lambda$ is

$$
\Lambda=E\left[\overline{\mathbf{Y}}(\tau) \overline{\mathbf{Y}}(\tau)^{H} \mid \overline{\mathbf{S}}(\tau)\right]
$$

$$
\begin{aligned}
& =E\left[(\overline{\mathbf{S}}(\tau) \overline{\mathbf{H}}(\tau)+\overline{\mathbf{N}}(\tau))(\overline{\mathbf{S}}(\tau) \overline{\mathbf{H}}(\tau)+\overline{\mathbf{N}}(\tau))^{H}\right] \\
& =E\left[\overline{\mathbf{S}}(\tau) \overline{\mathbf{H}}(\tau) \overline{\mathbf{H}}(\tau)^{H} \overline{\mathbf{S}}(\tau)^{H}+\overline{\mathbf{N}}(\tau) \overline{\mathbf{N}}(\tau)^{H}\right] \\
& =\overline{\mathbf{S}}(\tau) E\left(\overline{\mathbf{H}}(\tau) \overline{\mathbf{H}}(\tau)^{H}\right) \overline{\mathbf{S}}(\tau)^{H}+N_{r} \sigma_{n}^{2} \mathbf{I}_{N N_{t}(M+1)}
\end{aligned}
$$

At a certain time $t$, with (17), we have

$$
E\left\{H_{\mu \nu}^{p}(t) H_{\mu^{\prime} v^{\prime}}^{p^{\prime}}(t)\right\}=0 \quad\left(\mu \neq \mu^{\prime} \text { and } / \text { or } v \neq v^{\prime}\right)
$$

Set the $N \times N$ matrix $\Gamma_{F}$ as $\left[\Gamma_{F}\right]_{i j}=\rho_{F}(i-j)$. We have

$$
E\left[\overline{\mathbf{H}}(\tau) \overline{\mathbf{H}}(\tau)^{H}\right]=N_{r} \sigma_{h}^{2}\left(\Gamma_{F} \otimes \mathbf{I}_{N_{t}(M+1)}\right)
$$

and (31) can be recast into

$$
\Lambda=N_{r} \overline{\mathbf{S}}(\tau)\left(\Gamma_{F} \otimes \mathbf{I}_{N_{t}(M+1)}\right) \overline{\mathbf{S}}(\tau)^{H}+N_{r} \sigma_{n}^{2} \mathbf{I}_{N N_{t}(M+1)}
$$

Following a similar process as in time domain, we have

$$
\Lambda^{-1}=\frac{1}{N_{r} \sigma_{h}^{2}} \overline{\mathbf{S}}(\tau)\left[\left(\sigma_{n}^{2} \mathbf{I}_{N}+\Gamma_{F}\right)^{-1} \otimes \mathbf{I}_{N_{t}(M+1)}\right] \overline{\mathbf{S}}(\tau)^{H}
$$

and the frequency-domain decision metric of MSDD is

$$
\begin{aligned}
\eta^{\prime}= & -\operatorname{tr}\left\{\overline{\mathbf{Y}}(\tau)^{H} \overline{\mathbf{S}}(\tau)\left[\left(\sigma_{n}^{2} \mathbf{I}_{N}+\Gamma_{F}\right)^{-1} \otimes \mathbf{I}_{N_{t}(M+1)}\right]\right. \\
& \left.\cdot \overline{\mathbf{S}}(\tau)^{H} \overline{\mathbf{Y}}(\tau)\right\}
\end{aligned}
$$

Define

$$
f_{i j}=\left[-\left(\sigma_{n}^{2} \mathbf{I}_{N}+\Gamma_{F}\right)^{-1}\right]_{i j}
$$

we can finally get the simplified decision metric for diagonal constellations as

$$
\begin{aligned}
\eta= & \mathfrak{R}\left\{\sum_{n=0}^{M} \sum_{\mu=0}^{N_{t}-1} \sum_{v=0}^{N_{r}-1} \sum_{i=0}^{N-1} \sum_{k=i+1}^{N-1} f_{i k} \prod_{m=i}^{k-1} e^{j \frac{2 \pi u_{\mu} M+a_{m} a_{m}}{2^{(M+1)+1) N_{t}}}}\right. \\
& \left.\cdot y_{v}^{n N_{g}+g-i *}\left(N_{t} \tau+\mu\right) y_{v}^{n N_{g}+g-k}\left(N_{t} \tau+\mu\right)\right\}
\end{aligned}
$$

where

$$
\begin{aligned}
& s_{\mu}^{n N_{g}+g-i}\left(N_{t} \tau+\mu\right) s_{\mu}^{n N_{g}+g-k *}\left(N_{t} \tau+\mu\right) \\
& =\prod_{m=i}^{k-1} \exp \left(j \frac{2 \pi u_{\mu M+n} a_{m}}{2^{R(M+1) N_{t}}}\right)
\end{aligned}
$$

Thus $\hat{\mathbf{A}}$ is determined, if (38) is maximized.

4.3 Multiple-Symbol Differential Detection with Nondiagonal Constellations

As shown in above, the derivation of decision metrics (24) and (36) is not depended on whether diagonal or nondiagonal constellations are chosen. Therefore, DUSTFC with nondiagonal constellations is also able to be employed for the MSDD systems. The transmitted DUSTFC codewords can be estimated by utilizing decision metric (24) in time domain or decision metric (36) in frequency domain. And consequently the transmitted information sequence can be determined. 


\subsection{Computational Complexity}

It is obviously that the time-domain and frequency-domain MSDD for DUSTFC have the same computational complexity. The total computational complexity is a product of the following 2 parts: the complexity of calculating one decision metric, and the number of decision metrics required to be calculated and compared. We can observe from (26) and (38) that the complexity of calculating one decision metric is linear with $M+1, N_{t}, N_{r}$ and $\frac{N(N-1)}{2}$. On the other hand, because the number of possible values of $\mathbf{A}$ is $2^{R(M+1) N_{t}(N-1)}$, $2^{R(M+1) N_{t}(N-1)}$ decision metrics are required to be calculated and compared. Thus the complexity of the second part is exponential in $R,(M+1), N_{t}$ and $(N-1)$. Therefore, we know that the total complexity is mainly depended on $\underline{(M+1) N_{t} N_{r} N(N-1)} 2^{R(M+1) N_{t}(N-1)}$

Although the complexity of the first part can not be reduced, some suboptimal algorithms are proposed in [10], [15] and [16] to reduce the complexity of the second part. So that the computational complexity of the proposed system can be reduced by applying those algorithms.

\section{Simulation Results and Related Discussion}

In our simulations, a time-varying frequency selective Rayleigh fading channel model is used. A discrete exponential power-delay profile is used for simulating frequency selective fading. To focus on the effect of transmit diversity, here we set the number of receive antenna $N_{r}$ to 1 and the channels from different transmit antennas are assumed to be independent with each other. The number of OFDM subcarriers $N_{c}$ is 48 , the number of FFT points, which is equal to the data symbol period $T_{d}$, is 64 and the CP period $T_{g}$ is 16. The normalized maximum Doppler frequency $f_{D} T_{s}$ and the normalized root mean square delay spread $\sigma_{\tau} / T_{s}$ are set to various values for different channel environments. Since a transmit antenna is acted only once during transmitting a DUSTFC codeword in $N_{t} T_{s}$ period, we can define $N_{t} T_{s}$ as the effective sample period and accordingly $N_{t} f_{D} T_{s}$ is the effective normalized maximum Doppler frequency. We also set the data rate $R$ to 2 and the channel order $L$ to 5 . Note that since we want to consider the transmit diversity with constant bandwidth efficiency in this paper, $R$ is set to be constant. To keep $R$ constant, different constellation sizes are used for different $N_{t}$ and $M$, although using the same constellation size for bigger $N_{t}$ and/or $M$ (i.e. using constellation with lower $R$ ) can lead to better BER performance [3].

In the first example, the BER performances of frequency-domain MSDD for DUSTFC with different fading autocorrelations are compared. The conventional fading autocorrelation function (11) and the novel fading autocorrelation function (12) are used to calculate the decision metrics respectively. It is shown in Fig. 4 that the BER performance with decision metric using (12) is better than that with decision metric using (11), with any number of $N_{t}$ and $M$. Although such a performance gain is very small when

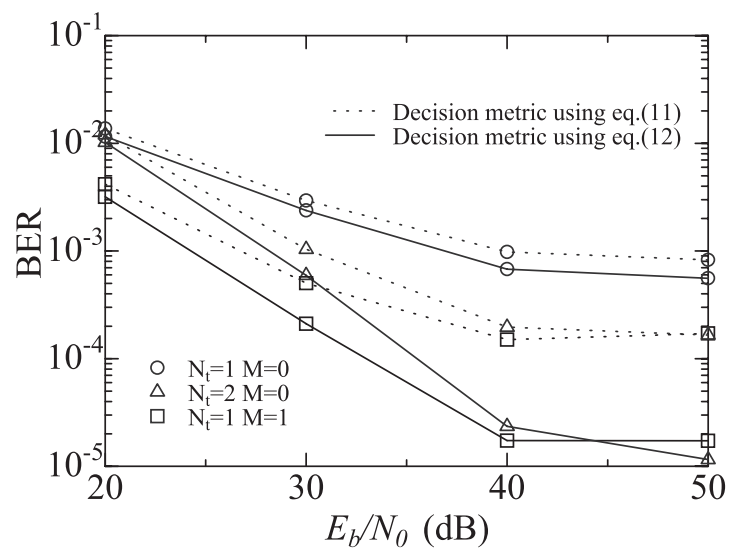

Fig. 4 The BER performance of frequency-domain MSDD for DUSTFC with different fading autocorrelations. $\left(f_{D} T_{s}=0.01, \sigma_{\tau} / T_{s}=0.01, N=\right.$ 4).

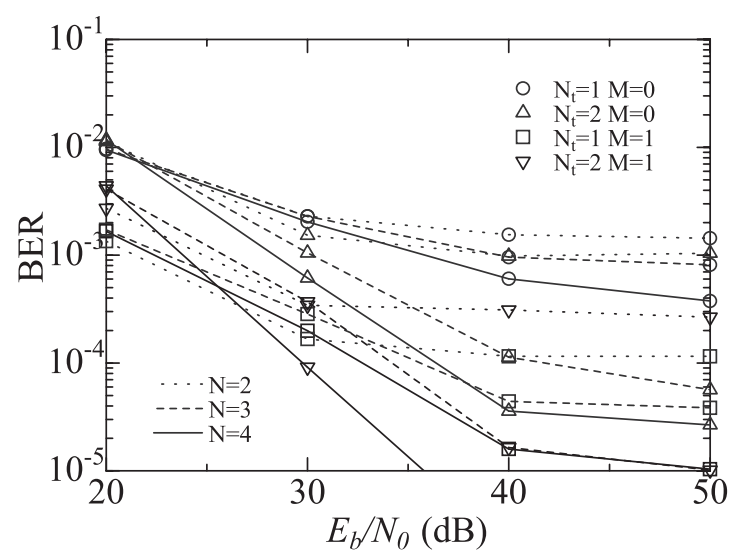

Fig. 5 The BER performance of time-domain MSDD for DUSTFC. $\left(f_{D} T_{s}=0.01, \sigma_{\tau} / T_{s}=0.01\right)$.

$N_{t}=1, M=0$ (no transmit diversity), considerable performance gain is yielded with applying transmit diversity. In this way, the effectiveness of novel fading autocorrelation is confirmed.

Figures 5, 6 and 7 show the BER performance of timedomain MSDD for DUSTFC. In Fig. 5, the BER performances at different signal-to-noise ratio (SNR) are given. In Figs. 6 and 7, the BER performances with various $\sigma_{\tau} / T_{s}$ and various $f_{D} T_{s}$ are compared respectively.

It is observed from Fig. 5, that at high SNR $\left(E_{b} / N_{0}>\right.$ $30 \mathrm{~dB}$ ), extending the observation interval enhances the BER performance and lowers the error floor, especially with multiple transmit antennas and/or multiple subcarriers in a group. Thus the validity of time-domain MSDD for DUSTFC is proved. It is also shown in this figure, that when $N=2$, using multiple transmit antennas has only a little benefit to the performance when $M=0$ and even leads to worse performance when $M=1$. It is because that when $N_{t}$ increases, $N_{t} f_{D} T_{s}$ also increases, which leads to an equivalent faster fading channel environment. And when the fading is fast, the influence of bigger $N_{t} f_{D} T_{s}$ is comparable with or even stronger than the diversity gain due to the increase of 


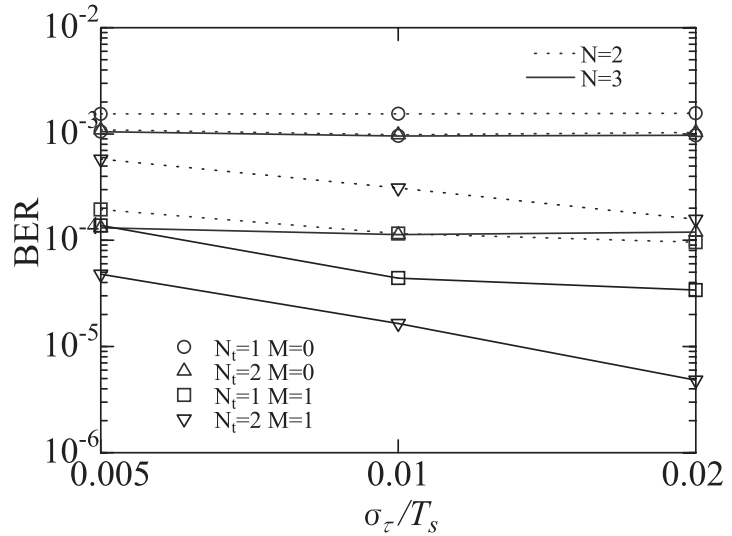

Fig. 6 The BER performance of time-domain MSDD for DUSTFC. $\left(f_{D} T_{s}=0.01, E_{b} / N_{0}=40 \mathrm{~dB}\right)$.

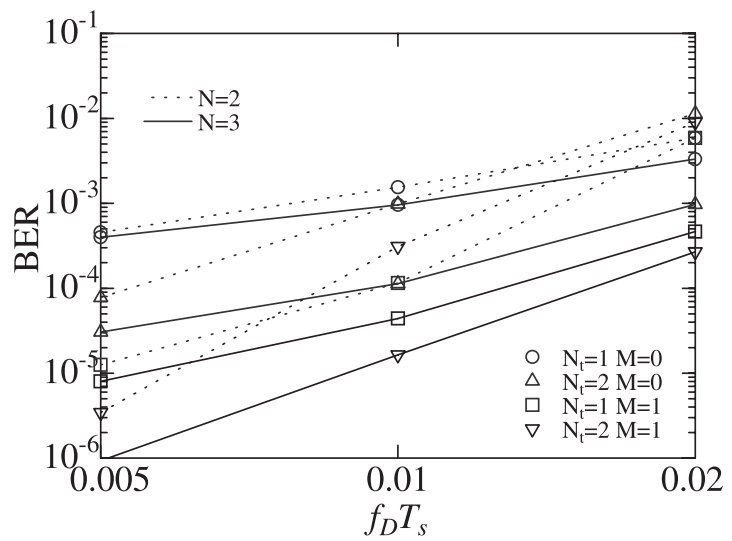

Fig. 7 The BER performance of time-domain MSDD for DUSTFC. $\left(\sigma_{\tau} / T_{s}=0.01, E_{b} / N_{0}=40 \mathrm{~dB}\right)$.

transmit antennas. When MSDD is used, significant performance gain can be observed by using multiple transmit antennas, since the effect of bigger $N_{t} f_{D} T_{s}$ is relieved by MSDD.

Figure 6 shows that when $M=0$ the BER performance hardly changes with different values of $\sigma_{\tau} / T_{s}$. That means the effect of frequency selective fading at the time domain is canceled by applying OFDM. And when $M=1$ the BER performance with bigger $\sigma_{\tau} / T_{s}$ is better. It is because that the frequency-domain autocorrelation becomes smaller with a bigger $\sigma_{\tau} / T_{s}$, consequently the correlation between replicas transmitted from subcarriers in a same group becomes smaller, and as a result more diversity gain can be obtained.

Figure 7 shows that the performance of time-domain MSDD becomes worse with the increase of $f_{D} T_{s}$. When $f_{D} T_{s}=0.02$, almost no benefit can be got by applying any kind of transmit diversity with $N=2$. But by extending the observation interval to $N=3$, considerable diversity gains can be obtained. It is also observed, that when $f_{D} T_{s}=$ 0.005 the benefit of using multiple transmit antennas can be confirmed with any observation interval length $N$.

The BER performance of frequency-domain MSDD for DUSTFC is shown in Figs. 8, 9 and 10. Similar with the

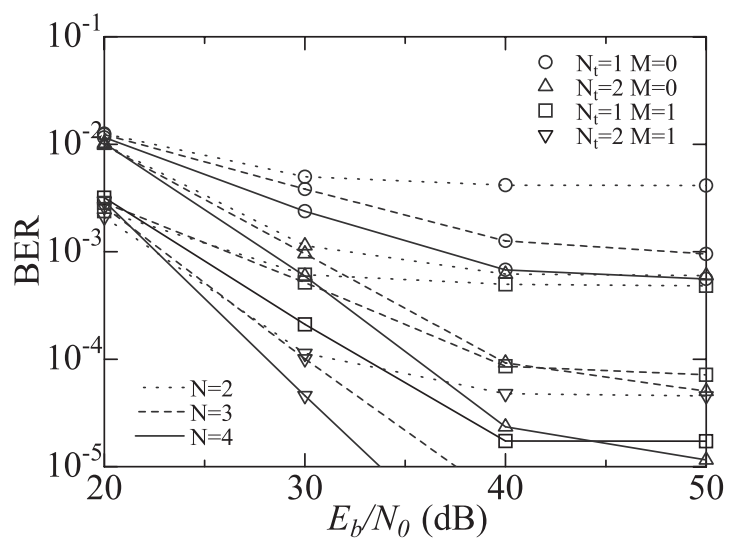

Fig. 8 The BER performance of frequency-domain MSDD for DUSTFC. $\left(f_{D} T_{s}=0.01, \sigma_{\tau} / T_{s}=0.01\right)$.

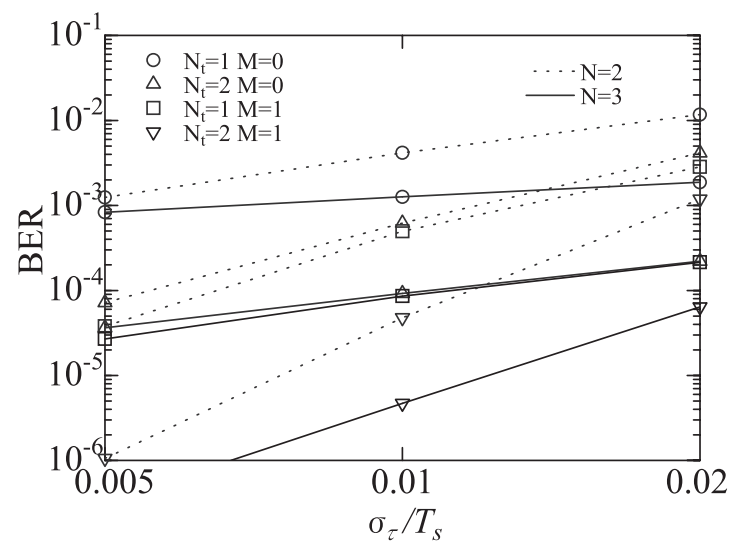

Fig. 9 The BER performance of frequency-domain MSDD for DUSTFC. $\left(f_{D} T_{s}=0.01, E_{b} / N_{0}=40 \mathrm{~dB}\right)$.

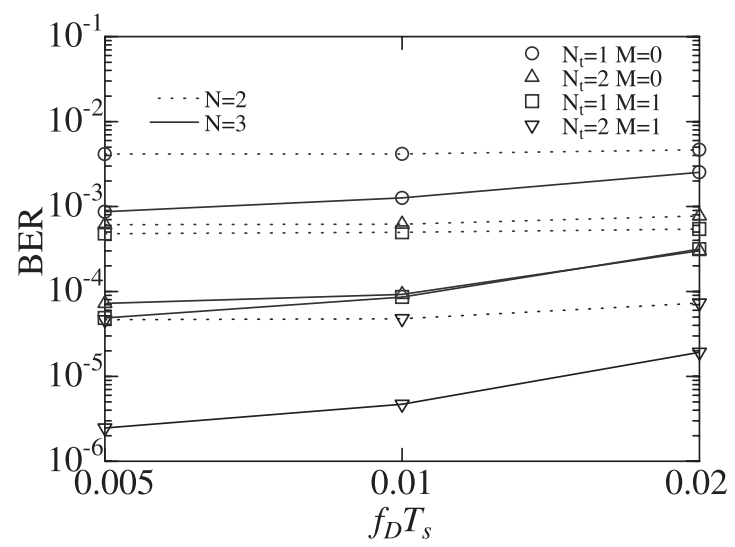

Fig. 10 The BER performance of frequency-domain MSDD for DUSTFC. $\left(\sigma_{\tau} / T_{s}=0.01, E_{b} / N_{0}=40 \mathrm{~dB}\right)$.

time-domain case, in Fig. 8, the BER performances at different signal-to-noise ratio (SNR) are given. In Figs. 9 and 10 , the BER performances with various $\sigma_{\tau} / T_{s}$ and various $f_{D} T_{s}$ are compared respectively.

We can observe from Fig. 8 that by extending the observation interval, the BER performance is improved and the 
error floor is lowered especially when the transmit diversity order is high, which proves the validity of frequency-domain MSDD for DUSTFC. It can also be observed that the benefit of using multiple transmit antennas can be confirmed with any observation interval length $N$, and at high SNR the BER performances with $N_{t}=1, M=1$ and $N_{t}=2, M=0$ are similar. It is because, unlike in the time-domain case, the change of $N_{t} f_{D} T_{s}$ itself has no influence on the frequencydomain detection.

Differently with the time-domain case, it can be observed from Fig. 9 that the performances with any number of $N_{t}$ and $M$ become better when $\sigma_{\tau} / T_{s}$ becomes lower. That means the effect of frequency selective fading becomes the main reason of error floor.

Figure 10 shows that the BER performance of frequency-domain MSDD becomes worse with the increase of $f_{D} T_{s}$, and the performance decreases relatively faster when $N=3$. It is because that bigger $f_{D} T_{s}$ leads to severer intercarrier interference (ICI) and the effect of ICI becomes larger when multiple subcarriers are considered together.

Comparing the BER performances with time-domain and frequency-domain MSDD, it can be easily observed that frequency-domain MSDD has better performance than the time-domain MSDD when $\sigma_{\tau} / T_{s}$ is low and vice versa. And we can also observe that frequency-domain MSDD is much less sensitive to various $f_{D} T_{s}$ than time-domain MSDD. When $f_{D} T_{S}$ is small, frequency-domain MSDD has worse performance than time-domain MSDD. But with the increase of $f_{D} T_{s}$, the performance of frequency-domain MSDD approaches and exceeds the performance of timedomain MSDD. Therefore, we can choose to apply whether time-domain or frequency-domain MSDD depending on various $\sigma_{\tau} / T_{s}$ and $f_{D} T_{s}$.

\section{Conclusion}

In this paper, MSDD was applied for DUSTFC over timevarying frequency selective fading multiple-input multipleoutput (MIMO) channels. Both the time and frequency domain MSDD were investigated, since the differential encoding of DUSTFC can be performed in time or frequency domain. After calculating the frequency-domain fading autocorrelation, decision metrics of MSDD were derived by considering the appropriate fading autocorrelation respectively in time and frequency domain. Simulation results demonstrated that applying both kinds of MSDD can lead to a significant performance gain. It was also observed that the diversity gain is enhanced by MSDD, especially in severe channel environments. That means full advantage of transmit diversity with DUSTFC can be taken by applying MSDD.

From the simulation results we can observe that error floors which may be caused by effect of ICI are still present in this system. Therefore, to suppress the ICI will be our main task in the future work. Also, the application of error correction is not considered in this paper. That is because in this paper, we want to focus on the benefit gained by apply- ing MSDD to DUSTFC. Choosing what kind of error correction method and how to combine the method with MSDD can affect the performance of the proposed system very differently. Thus the research of applying error correction to MSDD for DUSTFC is beyond the scope of this paper, and we will pursue such research in our subsequent work.

\section{References}

[1] V. Tarokh and H. Jafarkhani, "A differential detection scheme for transmit diversity," IEEE J. Sel. Areas Commun., vol.18, no.7, pp.1169-1174, July 2000.

[2] B.L. Hughes, "Differential space-time modulation," IEEE Trans. Inf Theory, vol.46, no.7, pp.2567-2578, Nov. 2000.

[3] B.M. Hochwald and W. Sweldens, "Differential unitary space-time modulation," IEEE Trans. Commun., vol.48, no.12, pp.2041-2052, Dec. 2000.

[4] S. Weinstein and P. Ebert, "Data transmission by frequency-division multiplexing using the discrete Fourier transform," IEEE Trans. Commun., vol.19, no.5, pp.628-634, Oct. 1971.

[5] J. Wang and K. Yao, "Differential unitary space-time-frequency coding for MIMO OFDM systems," Proc. 36th Asilomar Conf. Signals, Systems and Computers, pp.1867-1871, Pacific Grove, CA, Nov 2002

[6] Q. Ma, C. Tepedelenlioglu, and Z. Liu, "Differential space-timefrequency coded OFDM with maximum multipath diversity," IEEE Trans. Wireless Commun., vol.4, no.5, pp.2232-2243, Sept. 2005.

[7] Z. Liu, Y. Xin, and G.B. Giannakis, "Space-time-frequency block coded OFDM with subcarrier grouping and constellation precoding," Proc. ICASSP'02, vol.3, pp.2205-2208, May 2002.

[8] D. Divsalar and M.K. Simon, "Multiple-symbol differential detection of MPSK," IEEE Trans. Commun., vol.38, no.3, pp.300-308, March 1990.

[9] R. Schober and L.H.-J. Lampe, "Noncoherent receivers for differential space-time modulation," IEEE Trans. Commun., vol.50, no.5, pp.768-777, May 2002.

[10] S. Handa, Y. Okano, M. Liu, F. Sasamori, and S. Oshita, "Fast calculation algorithm and error performance of multiple-symbol differential detection over fading channels," IEICE Trans. Commun. vol.E86-B, no.3, pp.1050-1056, March 2003.

[11] Z. Jia, S. Handa, F. Sasamori, and S. Oshita, "Multiple-symbol differential detection for space-time block codes over fast Rayleigh fading channels," Proc. ICCT'06, Nov. 2006.

[12] F. Sasamori, S. Handa, and S. Oshita, "A simple method of BER calculation in DPSK/OFDM systems over fading channels," IEICE Trans. Fundamentals, vol.E88-A, no.1, pp.366-373, Jan. 2005.

[13] W.C. Jakes, ed., Microwave Mobile Communications, Wiley, New York, 1974.

[14] B. Hassibi and B.M. Hochwald, "Cayley differential unitary spacetime codes," IEEE Trans. Inf. Theory, vol.48, no.6, pp.1485-1503, June 2002.

[15] K.L. Clarkson, W. Sweldens, and A. Zheng, "Fast multiple-antenna differential decoding," IEEE Trans. Commun., vol.49, no.2, pp.253261, Feb. 2001.

[16] J.B. Anderson, "Limited search trellis decoding of convolutional codes," IEEE Trans. Inf. Theory, vol.35, no.5, pp.944-955, Sept. 1989. 


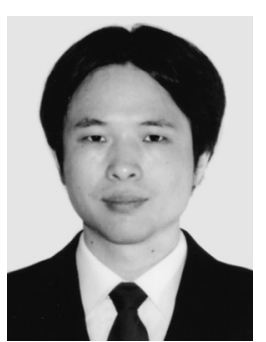

Ziyan Jia received the B.E. degree from Nanjing University of Posts and Telecommunications, China in 2004, and the M.E. degree from Shinshu University, Japan in 2007. He is currently pursuing the Dr. Eng. degree in the Department of Electrical and Electronic Engineering, Shinshu University. His current research interests include coding and modulation for mobile communications.

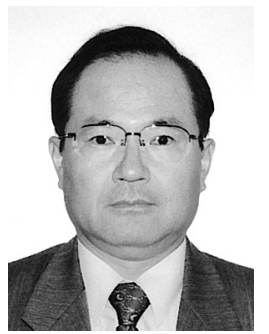

Shiro Handa received the B.E. and M.E. degrees from Shinshu University in 1978 and 1980 respectively, and the Dr. Eng. degree from Kobe University in 1988. From 1982 to 1988, he was a Research Associate at Kobe University. From 1988 to 1994, he was with Nagano National College of Technology. He has been with the Department of Electrical and Electronic Engineering, Shinshu University, since 1994 as an Associate Professor and since 2005 as a Professor. In 1996, he was at the University of California Davis, as a visiting researcher. His research interests include satellite and mobile communication systems, modulation and coding, and data compression. He is a member of IEEE and SITA.

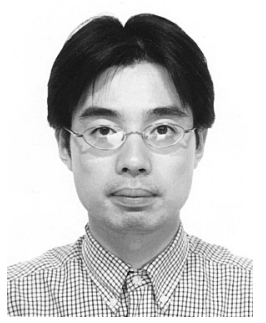

Fumihito Sasamori received the B.E., M.E and Dr.Eng. Degrees from Waseda University, Tokyo in 1994, 1996 and 2000, respectively. Since 2000 he has been with the Department of Electrical and Electronic Engineering, Shinshu University, first as a Research Associate and since 2006 as an Associate Professor. His current research interests include digital mobile communication systems. He received the IEICE Young Engineer Award in 2000. He is a member of IEEE.

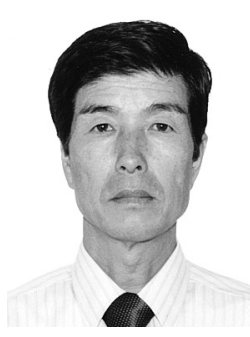

Shinjiro Oshita received the B.E. degree from Osaka Electro Communication University in 1967, the M.E. and Dr.Eng. degrees from Osaka University, Japan in 1970 and 1973, respectively. Since 1973, he has been with Shinshu University, Nagano, Japan, where he is currently a Professor in the Department of Electrical and Electronic Engineering. His research interests include intelligent CAI and communications theory. He is a member of IEEE and Japanese Society for Information and System in

Education. 